\title{
Measurement technique for bidirectional four sensor rotary encoder
}

\begin{abstract}
Measurement and monitoring of fluid flow are considered as crucial issues in industry and domestic applications especially for the water measurement. Numerous approach based on different physical principles have been proposed such as ultrasonic, electromagnetic, and differential pressure systems which each one of them requires its own particular measurement technique and algorithm to compute the necessary information such as flow speed, consumption and flow direction. However, there are some weaknesses in their measurement techniques including complexity and power consumption in their implementation. Therefore, in this paper, a measurement technique based on four sensor rotary encoder is presented. The measurement technique is simple and able to measure the speed, number of rotation and recognize the direction of the rotation. Furthermore, in the proposed measurement technique the error of the system is detectable in which debugging the system is required expensive equipment and long time for the conventional rotary encoder system. The functionality and performance of the measurement technique have been evaluated and found that the proposed technique is able to accurately measure the flow speed, the number of rotation, identify the direction of rotation and recognize the error occurred.
\end{abstract}

Keyword: Bidirectional rotary encoder; Rotary encoder measurement algorithm; Fluid measurement 